\title{
Active surface area approximation in a lead-acid cell for optimal performance in renewable energy systems
}

\author{
Angelique Janse van Rensburg*, George van Schoor*, \\ Pieter A. van Vuuren** \\ *Unit for Energy Systems, North-West University, Potchefstroom, South Africa \\ (e-mail:20160135@nwu.ac.za,george.vanschoor@nwu.ac.za) \\ ** School of Electrical, Electronic and Computer Engineering, North-West University, \\ Potchefstroom, South Africa (e-mail: pieter.vanvuuren@nwu.ac.za)
}

\begin{abstract}
In a renewable energy system, lead-acid batteries are required to last as long as possible whilst providing deep discharges and are usually charged in an intermittent manner. This type of operation is severely detrimental to the electrodes and optimal performance cannot be guaranteed. Correct operation can be achieved if a charge controller receives accurate information about the state of the battery and its cells. Accurate approximation of the available active surface area is essential to optimal performance since it determines the overall capacity and reaction rates of various secondary processes. This paper improves on the current understanding of the active surface area in a lead-acid cell during discharge by comparing three approximation methods. Two methods use the state-of-charge based on porosity whilst the other method uses the charge per unit volume to determine the remaining capacity. These three methods are used in a comprehensive macro-homogeneous electrochemical model which accounts for initial temperature differences. Reported experimental behaviour is used to evaluate each method and recommendations are made regarding the selection of associated parameter values. The approximation method by Cugnet et al. (2009) is the most suitable because it always results in a concave morphology. The current work can aid in the development of a health-conscious battery management system.
\end{abstract}

Keywords: reliability, energy efficiency, lead-acid battery, electrochemical model, active surface area, electrode morphology

\section{INTRODUCTION}

The global energy crisis is one of the biggest challenges humankind has ever had to face. This crisis is a substantial driving force behind research and development in alternative energy technologies (Liu et al., 2012). Renewable energy originates from inexhaustible sources like the sun or wind sources which are inherently variable and intermittent (Goodenough et al., 2007). By integrating some form of energy storage into a renewable energy system, a reliable supply of energy can be ensured (Ibrahim et al., 2008).

Batteries are easy to use, capable of high energy densities and do not depend on geographic location. Apart from renewable energy systems and electric vehicles, battery energy storage is also widely used in telecommunications applications (Pavlov, 2011). Lead-acid battery (LAB) technology is currently the most popular method of battery energy storage because it is affordable, readily available and easy to recycle (Kaiser, 2007). These are just some of the reasons why the lead-acid battery industry accounted for a global market share of US\$ 60.3 billion in 2010 (Liu et al., 2012).

The use of advanced designs like valve-regulated lead-acid (VRLA) batteries is only expected to grow. This upward trend is despite that, in renewable energy applications, more than a third of VRLA batteries fail long before their intended design life (DeAnda et al., 2004). A post-failure investigation can be performed to determine exactly why the battery has failed but it is less expensive and easier to simply buy a new one. Over the long term however, having to replace the battery often amounts to unnecessary and high operating costs (Kaiser, 2007). A health-conscious battery management system (BMS) with intelligent charge control can be used to address this lifetime issue and improve the reliability of VRLA batteries.
When used incorrectly, stress factors cause the battery to degrade faster than it otherwise would. These factors include, among others, overcharging, overheating and cycling. How severely the factors stress the battery is directly related to the amount of available active surface area. Battery degradation consists of several different damage mechanisms: corrosion of the positive grid, irreversible sulphation, shedding, water loss, active material degradation and electrolyte stratification (Andersson, 2006). Irreversible sulphation and corrosion insulate the active material from electrolyte and reduce the available active surface area. The net result is an overall loss of battery capacity (Bindner et al., 2005).

It should be clear that the active surface area plays a crucial role in determining the overall capacity of a lead-acid cell. Since capacity is the most significant property a BMS has to calculate (Codecà et al., 2008), it needs to take this area into account. As such, the active surface area is the focus of this paper and it is emphasized in bold italic text in Fig. 1. As can be seen, the current work forms part of a bigger research project. Phase 1 of the project consists of developing and validating a multi-scale model of a VRLA battery.

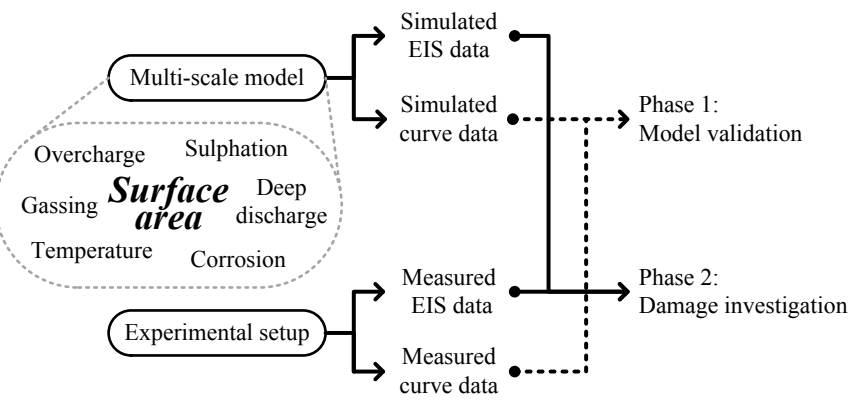

Fig. 1. Illustration of current work as part of research project 
The validated model and data from electrochemical impedance spectroscopy (EIS) experiments will then be used to investigate damage mechanisms in Phase 2.

If the active surface area is approximated to be larger than it actually is, a lead-acid cell might be discharged past its rated depth-of-discharge. This results in electrode damage by irreversible sulphation. On the other hand, if the active surface area is approximated to be smaller than it actually is, the full discharge capacity cannot be utilized. In either case, the lead-acid cell cannot deliver energy in the most efficient and reliable manner possible.

The aim here is to evaluate various methods of approximating the active surface area. This is achieved by selecting three candidates from the relevant literature and comparing their outputs with experimentally reported behaviour. The current work enables quicker selection of a suitable method to approximate the active surface area and the associated parameter values. In this way, the time required by the modelling process is decreased.

The next section explains the electrochemical model used in the comparison. Section 3 provides the details of the approximation methods under consideration. The numerical solution of the complete model is discussed in Section 4 followed by the simulation results in Section 5. The findings are discussed and the work concluded in Section 6.

\section{ELECTROCHEMICAL MODEL}

Battery models based on fundamental electrochemical principles can be very complex but they are more accurate than any other modelling approach (Jongerden and Haverkort, 2008). The current model makes use of volumeaveraged quantities and considers an electrode to be a solid matrix with concentrated electrolyte filling the pores. This approach is commonly known as the macro-homogeneous approach (Whitaker, 1986). For detailed information on the macro-homogeneous approach, refer to the work by Newman and Tiedemann (1975).

Consider Fig. 2 which shows a lead-acid cell consisting of four regions: the positive electrode $\left(\mathrm{PbO}_{2}\right)$, an electrolyte reservoir, a micro-porous separator and the negative electrode $(\mathrm{Pb})$. Even though the cell is modelled in 1D with distributed quantities in the direction of the $x$-axis, Fig. 2 shows the cell in 2D for clarity. The $y$-axis direction is parallel to the cell's height in $2 \mathrm{D}$ models. The electrolyte $\left(\mathrm{H}_{2} \mathrm{SO}_{4}\right)$ is considered binary and dissociates fully into its respective ions.

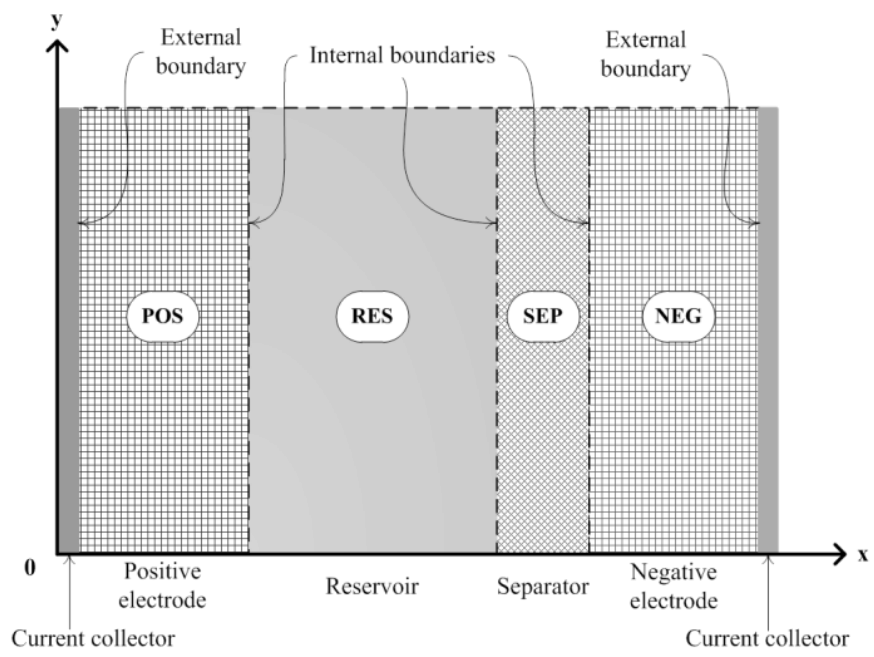

Fig. 2. Illustration of a unit cell in $2 \mathrm{D}$
The current collectors in Fig. 2 represent the centre of each electrode - only half of an electrode is shown in the unit cell. The two external boundaries therefore denote a plane of symmetry in the electrodes. This unit cell is repeated to form a stack of cells in an actual battery. The stoichiometry of the primary reduction-oxidation reaction is given by

$$
\mathrm{PbO}_{2(\mathrm{~s})}+\mathrm{HSO}_{4}^{-}+3 \mathrm{H}^{+}+2 \mathrm{e}^{-} \underset{\text { charge }}{\stackrel{\text { discharge }}{\rightleftarrows}} \mathrm{PbSO}_{4(\mathrm{~s})}+2 \mathrm{H}_{2} \mathrm{O}
$$

for the positive $\mathrm{PbO}_{2}$ electrode and

$$
\mathrm{Pb}_{(\mathrm{s})}+\mathrm{HSO}_{4}^{-} \underset{\text { charge }}{\stackrel{\text { discharge }}{\rightleftarrows}} \mathrm{PbSO}_{4(\mathrm{~s})}+\mathrm{H}^{+}+2 \mathrm{e}^{-}
$$

accounts for the reaction at the negative $\mathrm{Pb}$ electrode.

\subsection{Effects of temperature and concentration}

Even though conditions are considered to be isothermal, adjustments have been included to account for initial temperature differences. The dependence of the reference concentration on cell temperature as given by Bode (1977) is used in this model. According to $\mathrm{Gu}$ et al. (1987), the diffusion coefficient of the electrolyte depends on the concentration of the electrolyte and on the cell's temperature. This dependence is included in the current work.

Tenno et al. (2001) account for changes in the reference transfer current density due to the initial temperature difference. The authors use an equation containing the activation energy - it is also used here. The effect of the concentration's molality on the thermodynamic potential of an electrode is given by Bode (1977). It is used in the same manner as Cugnet et al. (2009) to determine the potentials of the positive and negative electrodes respectively. The conductivity of the electrolyte is given by Nguyen et al. (1990) and the conductivity of lead by Bode (1977).

\subsection{Governing equations for discharge}

The governing equations for the discharge behaviour of the lead-acid cell were developed from work by Gu et al. (1987), Bernardi and Carpenter (1995) and Cugnet et al. (2009). For each electrode, there are five unknown variables in the governing equations: (i) porosity of the electrode $\varepsilon$, (ii) potential of the pore electrolyte $\phi_{l}$, (iii) potential in the solid phase $\phi_{s},(i v)$ concentration of the sulphuric acid $c$, and $(v)$ the superficial current density in the liquid phase $i_{l}$.

Similar to the work by Cugnet et al. (2009) and Bernardi and Carpenter (1995), the governing equations were simplified using parameters $K_{i}$ as explained in the authors' respective works. The other symbols used in the following equations are listed at the end of this paper.

The porosity decreases as $\mathrm{Pb}$ and $\mathrm{PbO}_{2}$ are converted to $\mathrm{PbSO}_{4}$ which insulates the electrodes and decreases the available active surface area. A material balance equation accounts for this porosity variation over time and is given by

$$
\frac{\partial \varepsilon}{\partial t}+K_{1} \frac{\partial i_{l}}{\partial x}=0
$$

in the solid phase. Ohm's Law is modified for use in the liquid phase as

$$
\frac{i_{l}}{\varepsilon^{\mathrm{ex} 1} \kappa}+\frac{\partial \phi_{l}}{\partial x}+K_{2} \frac{\partial \ln c}{\partial x}=0
$$

to show that the current density in the electrolyte $i_{l}$ is driven by electric and chemical potential gradients. Ohm's Law applied to the solid matrix is given by

$i_{l}-\varepsilon^{\mathrm{exm} 1} \sigma_{\mathrm{PbO}_{2}} \frac{\partial \phi_{s}}{\partial x}-i_{a p p}=0$ 
where $i_{a p p}$ is the applied discharge current density in $\mathrm{A} / \mathrm{cm}^{2}$. The material balance equation which accounts for changes in the pore electrolyte concentration is given by

$$
\varepsilon \frac{\partial c}{\partial t}+K_{3} i_{l} \frac{\partial c}{\partial x}-\frac{\partial}{\partial x}\left(\varepsilon^{\mathrm{ex} 1} D_{l} \frac{\partial c}{\partial x}\right)+\left(K_{4} c+K_{5}\right) \frac{\partial i_{l}}{\partial x}=0
$$

which includes mass transport due to diffusion, migration and convection. The electrode reaction is given by

$$
\frac{\partial i_{l}}{\partial x}-a_{v} i_{l o c}-C_{d l} a_{\max }\left(\frac{\partial \phi_{s}}{\partial t}-\frac{\partial \phi_{l}}{\partial t}\right)=0
$$

with $C_{d l}$ called the double-layer capacitance. The variable $a_{v}$ is the available active surface area in $\mathrm{cm}^{2} / \mathrm{cm}^{3}$ and $i_{l o c}$ is the local current density at the electrode-electrolyte interface. Equations (1) to (4) are not explained in detail since the focus here is on the active surface area used in (5). Different ways of calculating this area are now discussed.

\section{ACTIVE SURFACE AREA APPROXIMATION}

The active surface area is the true area of active material in contact with electrolyte and can be much greater than the geometric electrode area. This contact interface between the electrode and the electrolyte is called the electrical doublelayer or EDL. In existing macro-homogeneous models, the available active surface area is usually approximated using an expression based on the state-of-charge (SOC). The cell's SOC can be calculated using either the electrode porosity $\varepsilon$ as done by Kashkooli et al. (2013) or the charge per unit volume $Q$ in $\mathrm{C} / \mathrm{cm}^{3}$ as done by Kim and Hong (1999).

The SOC using electrode porosity during discharge of a leadacid cell is given by

$$
\mathrm{SOC}=\frac{\varepsilon-\varepsilon_{0}}{\varepsilon_{\max }-\varepsilon_{0}}
$$

whereas the SOC using charge per unit volume is given by

$$
\mathrm{SOC}=\frac{1}{Q_{\max }} \int_{0}^{t}\left(\nabla \cdot i_{l}\right) d t
$$

which is commonly known as Coulomb-counting. $Q_{\max }$ is the theoretical maximum capacity of the electrode under consideration and depends on the volume fraction of the solid phase (Tiedemann and Newman, 1975). For each electrode, the volume fraction of the solid phase $\varepsilon_{s}$ can be determined by subtracting the porosity $\varepsilon$ and any other fraction of inert material. The available volume fraction of an electrode is then given by

$$
\varepsilon_{\mathrm{s}}=1-\varepsilon_{\text {max }}-\varepsilon_{\text {inert }}
$$

where $\varepsilon_{\text {inert }}$ is the volume fraction of inert material in the electrode. Using Faraday's First Law of Electrolysis and accounting for porosity, the maximum capacity of an electrode of material $\mathrm{M}$ is calculated using

$Q_{\max }=\left(\frac{2 F \rho_{\mathrm{M}}}{\mathrm{MW}_{\mathrm{M}}}\right) \cdot \varepsilon_{\mathrm{s}}$

with $F$ the Faraday constant of $96,485.3365(\mathrm{~A} \cdot \mathrm{s}) / \mathrm{mol}$. The SOC from either (6) or (7) is then used to approximate the available active surface area during discharge as

$a_{v}=a_{\max } \mathrm{SOC}^{\zeta}$

in $\mathrm{cm}^{2} / \mathrm{cm}^{3}$. The parameter $\zeta$ in (10) represents electrode morphology and its value differs between models. The active surface area can now be used in (5) to determine the interfacial current density with $i_{l o c}$ given by the ButlerVolmer Law in (11). $i_{\text {loc }}=i_{0}\left(\frac{c}{c_{\text {ref }}}\right)^{\gamma}\left[\exp \left(\frac{\alpha_{a} F \eta}{R T_{\text {cell }}}\right)-\exp \left(-\frac{\alpha_{c} F \eta}{R T_{\text {cell }}}\right)\right]$

The electrode reaction order is represented by $\gamma$ in (11) and its value can only be determined experimentally. The complete expression for the local current density is given by

$$
\begin{aligned}
a_{v} i_{\text {loc }}= & a_{\max } \mathrm{SOC}^{\zeta} . \\
& i_{0}\left(\frac{c}{c_{\text {ref }}}\right)^{\gamma}\left[\exp \left(\frac{\alpha_{a} F \eta}{R T_{\text {cell }}}\right)-\exp \left(-\frac{\alpha_{c} F \eta}{R T_{\text {cell }}}\right)\right]
\end{aligned}
$$

with the SOC as in (6) or (7). Cugnet et al. (2009) calculate the local current density by introducing a new exponential expression as shown in braces in (13). In this equation, the SOC is still derived from porosity but it is used differently.

$$
\begin{aligned}
a_{v} i_{l o c}= & a_{\max }\left\{1-\exp \left[-\zeta\left(\frac{\varepsilon-\varepsilon_{0}}{\varepsilon_{\max }-\varepsilon_{0}}\right)\right]\right\} . \\
\frac{i_{0} \rho_{d}}{c_{r e f}^{\gamma}} & \left.\exp \left(\frac{\alpha_{a} F \eta}{R T_{\text {cell }}}\right)-\exp \left(-\frac{\alpha_{c} F \eta}{R T_{\text {cell }}}\right)\right]
\end{aligned}
$$

The authors also introduce another empirical parameter $\rho_{d}$ but exclude the varying electrolyte concentration $c$. The reservoir and separator region do not consist of electrode material and therefore, do not have active surface areas. These two regions have governing equations similar to the electrode regions but their respective porosities do not vary with time. The reader is referred to the work of Cugnet et al. (2009) for the complete sets of equations.

The active area approximations as used by Kashkooli et al. (2013), Cugnet et al. (2009) and Kim and Hong (1999) will be used in (5) and the current model simulated. Their approximations will be denoted as Kashkooli, Cugnet and Kim from this point forward. Value selections for the reaction order $\gamma$ and electrode morphology $\zeta$ will also be discussed in Section 5 with the simulation results. The remaining parameter values are the same as those used in Cugnet et al. (2009).

\section{NUMERICAL SOLUTION}

COMSOL Multiphysics ${ }^{\circledR}$ was used to solve the governing equations for each region during the discharge of a lead-acid cell. It simply takes too long to compute the time integral as shown in (7). Instead, the equation was converted to

$$
\frac{\partial \mathrm{SOC}}{\partial t}= \pm \frac{\nabla \cdot i_{l}}{Q_{\max }}
$$

and programmed as a partial differential equation to obtain the SOC. The speed-up in computation time was exponential without negatively affecting the solution's accuracy.

Initial conditions were chosen to represent a lead-acid cell at the beginning of discharge with the SOC at $100 \%$. The electrolyte concentration $c$ is considered uniform throughout the cell. The porosity $\varepsilon$ is initially considered uniform for each electrode and has the maximum value as given by Cugnet et al. (2009). The initial solid potential of the positive electrode is the difference between the thermodynamic potentials of the electrodes. The negative electrode has an initial solid potential of $0 \mathrm{~V}$. The solid potential is nonexistent in the RES and SEP regions. Initial values for other variables are set to zero since they are calculated by the simulation.

External boundary conditions were chosen to represent a sealed unit. The discharge current is applied to the left 
external boundary and the right external boundary corresponds to electric ground. At these two external boundaries, there is no pore electrolyte and therefore, no superficial current density. Internal boundary conditions were chosen to ensure continuity of both flux and current density across each interface. Refer to the work of Gu et al. (1987) and Cugnet et al. (2009) for detailed explanations on boundary and initial conditions used in the macrohomogeneous approach.

\section{SIMULATION RESULTS}

To ensure that the current work would be simulated correctly in COMSOL Multiphysics ${ }^{\circledR}$, the complete simulations by Kashkooli et al. (2013), Cugnet et al. (2009) and Kim and Hong (1999) were repeated. The results compared well with those of the respective authors though they are not published in this paper. The models by these authors differ when it comes to material balance equations and temperature effects. To ensure an objective comparison between the different ways of approximating the active surface area, the generic model described in Section 2 was used in the current work. Thus, only the active surface area approximations are different from one simulation to the next.

The following simulations each completed in a matter of seconds on a laptop computer with an Intel i7-2670QM processor and $16 \mathrm{~GB}$ of RAM. Presented first is a C/20 discharge with $1 \mathrm{~h}$ rest at $20^{\circ} \mathrm{C}$ of a cell from a battery rated at $60 \mathrm{Ah}$. The discharge current density based on total plate area of the cell equates to $-14.245 \mathrm{~A} / \mathrm{m}^{2}$. An initial step size of $1 \mathrm{~s}$ and a maximum step size of $500 \mathrm{~s}$ were used. Original parameter values as chosen by the respective authors were used.

Cell voltages predicted by Kashkooli, Cugnet and Kim are shown in Fig. 3. Note that the minimum voltage from Kashkooli is lower than the cut-off voltage of $1.75 \mathrm{~V}$. In reality, this cell would have provided less discharge time.

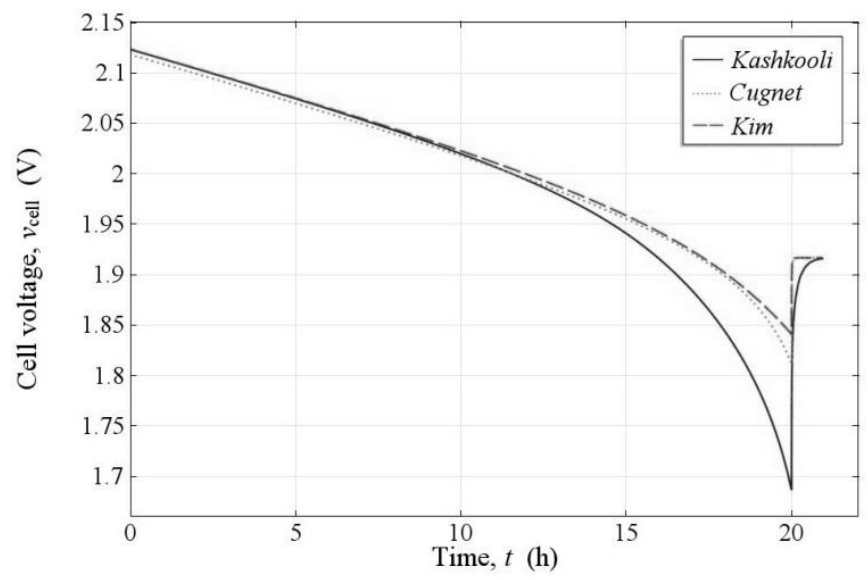

Fig. 3. Simulated cell voltage during $\mathrm{C} / 20$ discharge and $1 \mathrm{~h}$ rest at $20{ }^{\circ} \mathrm{C}$ using original parameter values

The morphology of the positive electrode over time is illustrated in Fig. 4. The $x$-axis is the SOC of the electrode based on porosity or charge per unit volume. The $y$-axis is the active surface area normalized using the maximum active surface area. The morphology predicted by Cugnet is problematic since it estimates that the initial active surface area is only $40 \%$ of the maximum value. The authors of this approximation method do mention that some parameters have to be calibrated with experimental data. In the current work, it was found that a $100 \%$ initial active surface area could be predicted if the morphology parameter was changed to 6 .

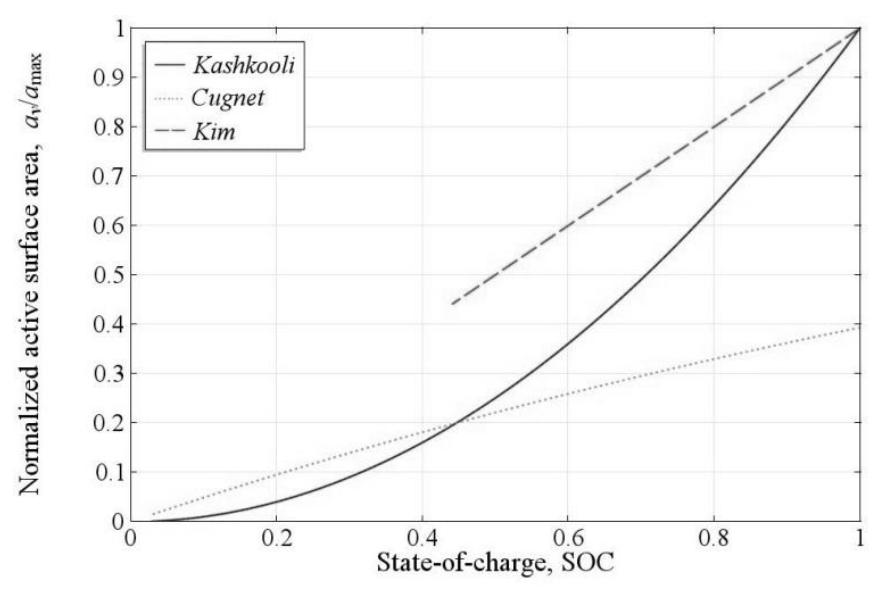

Fig. 4. Simulated morphology for POS during $\mathrm{C} / 20$ discharge at 20 ${ }^{\circ} \mathrm{C}$ using original parameter values

The approximation by Kim also presents an issue. Its final SOC value depends on the maximum electrode capacity calculated using (9). This equation accounts for the initial volume fraction of solid material but not for the final volume fraction. Since the maximum and minimum porosity values are available, it can be used to calculate a better estimate for the maximum electrode capacity. Tiedemann and Newman (1975) calculates the change in solid volume fraction $\Delta \varepsilon_{s}$ using

$$
\Delta \varepsilon_{\mathrm{s}}=\left(1-\varepsilon_{0}-\varepsilon_{\text {inert }}\right)-\left(1-\varepsilon_{\max }-\varepsilon_{\text {inert }}\right)=\varepsilon_{\text {max }}-\varepsilon_{0}
$$

and substitutes it into (9) to calculate $Q_{\max }$. The modified parameters for Cugnet and Kim can be found in Table 1 and Table 2 respectively. The approximation by Kashkooli uses values of 2 for $\zeta$ and 0 for $\gamma$ which were not modified from the authors' original values.

Table 1: Modified parameters for Cugnet

\begin{tabular}{clcc} 
Symbol & Name & Original & Modified \\
\hline$\zeta$ & Morphology & 0.5 & 6 \\
$\gamma$ & Reaction order & 0 & - \\
$\rho_{d}$ & Empirical parameter & 1 & 0.2
\end{tabular}

Table 2: Modified parameters for Kim

\begin{tabular}{clcc} 
Symbol & Name & Original & Modified \\
\hline$\zeta$ & Morphology & 1 & - \\
$\gamma$ & Reaction order & 1.5 & - \\
$\Delta \varepsilon_{\text {s,pos }}$ & Capacity factor for POS & 0.33 & 0.1834 \\
$\Delta \varepsilon_{\text {s,neg }}$ & Capacity factor for NEG & 0.29 & 0.2234 \\
$Q_{\text {max,pos }}$ & Maximum capacity for POS & $2,600 \mathrm{C} / \mathrm{cm}^{3}$ & $1,500 \mathrm{C} / \mathrm{cm}^{3}$ \\
$Q_{\text {max,neg }}$ & Maximum capacity for NEG & $3,100 \mathrm{C} / \mathrm{cm}^{3}$ & $2,400 \mathrm{C} / \mathrm{cm}^{3}$
\end{tabular}

Fig. 5 and Fig. 6 show the predicted cell voltages and morphology over time using these modified parameter values for the same discharge simulation.

The cell voltage predicted using Kim is much lower now since the maximum capacity for each electrode has been lowered. Morphologies for the positive electrode as predicted by Cugnet and Kim do not present the same problems experienced using the authors' original parameter values. The values for the reaction order $\gamma$ have not been modified from the original values since they can only be accurately determined with experimental data according to Ekdunge and Simonsson (1985). 


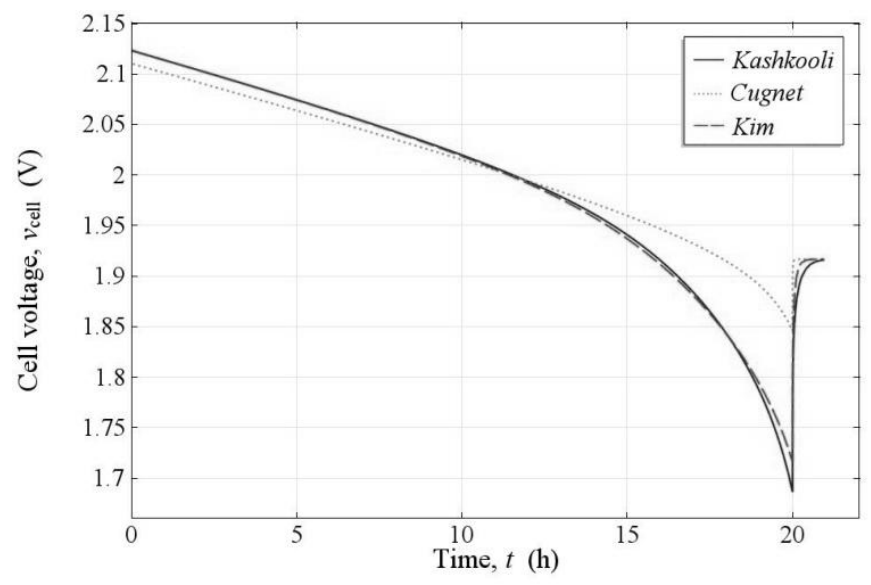

Fig. 5. Simulated cell voltage during $\mathrm{C} / 20$ discharge and $1 \mathrm{~h}$ rest at $20^{\circ} \mathrm{C}$ using modified parameter values

Fig. 6 clearly shows how the cell starts with the maximum surface area for each approximation method. Since the simulations using Kashkooli, Cugnet and Kim are initialized in a more objective manner with the modified parameter values, they will be used from this point forward.

Similar final concentrations and porosities for the different regions were predicted by Kashkooli, Cugnet and Kim. This allows for the assumption that the simulation results enable a fair comparison because the active surface area output only varies according to the chosen approximation method.

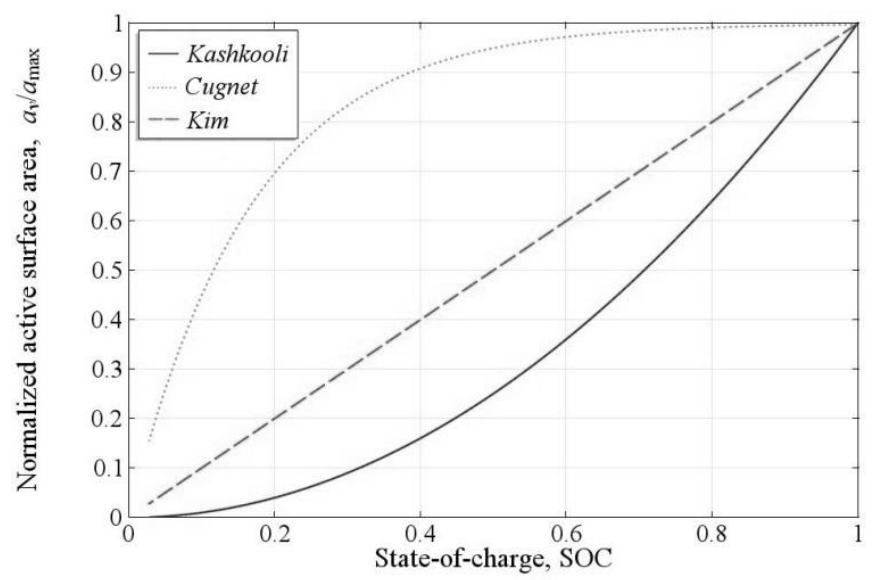

Fig. 6. Simulated morphology for POS during $\mathrm{C} / 20$ discharge at 20 ${ }^{\circ} \mathrm{C}$ using modified parameter values

Results for a high-rate discharge at $10 \mathrm{C}$ are now presented. The cell discharge current density equates to $-2849 \mathrm{~A} / \mathrm{m}^{2}$ at this C-rate. The simulations were set to stop the discharge when a cut-off voltage of $1.75 \mathrm{~V}$ was reached. The predicted cell voltages over time are shown in Fig. 7.

This result depicts how a charge controller might prematurely end the discharge if it estimated the active surface area smaller than it actually is. In high-rate applications, an error of $30 \mathrm{~s}$ in the discharge time can be disastrous. The importance of correctly approximating the active surface area should be clear at this point.

In the final simulation, an intermittent discharge is applied to the lead-acid cell. It was performed over $20 \mathrm{~h}$ at an applied current density of $-14.245 \mathrm{~A} / \mathrm{m}^{2}$ which is the same as the previous $\mathrm{C} / 20$ discharge. The only difference is an intermittent rest period of $10 \mathrm{~s}$ followed by discharging.

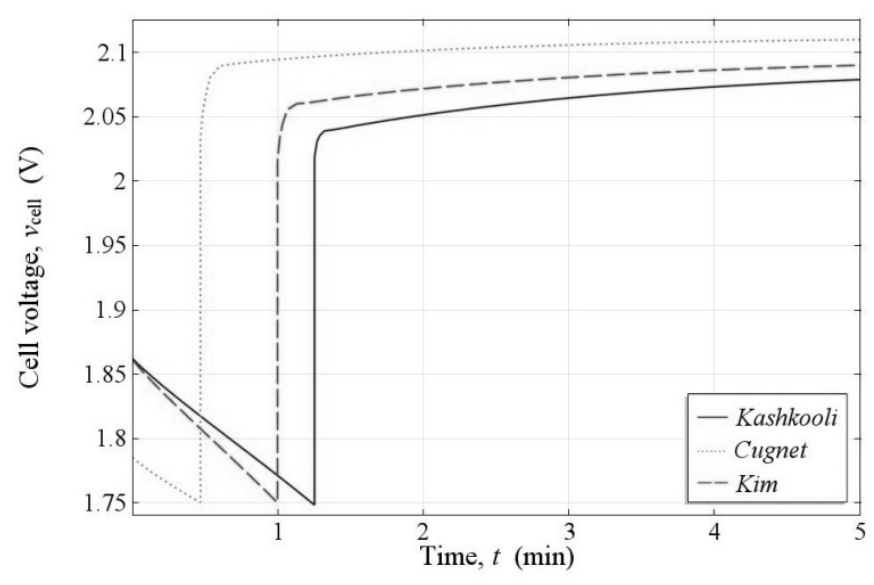

Fig. 7. Simulated cell voltage during $10 \mathrm{C}$ discharge at $20^{\circ} \mathrm{C}$ using modified parameter values

Fig. 8 shows how much slower the capacity and active surface area decrease for the same time period when compared to Fig. 6. The idea of extracting more energy from a battery by letting it rest intermittently is not new (Ng et al., 2007). This last result supports it and warrants further investigation of the phenomenon.

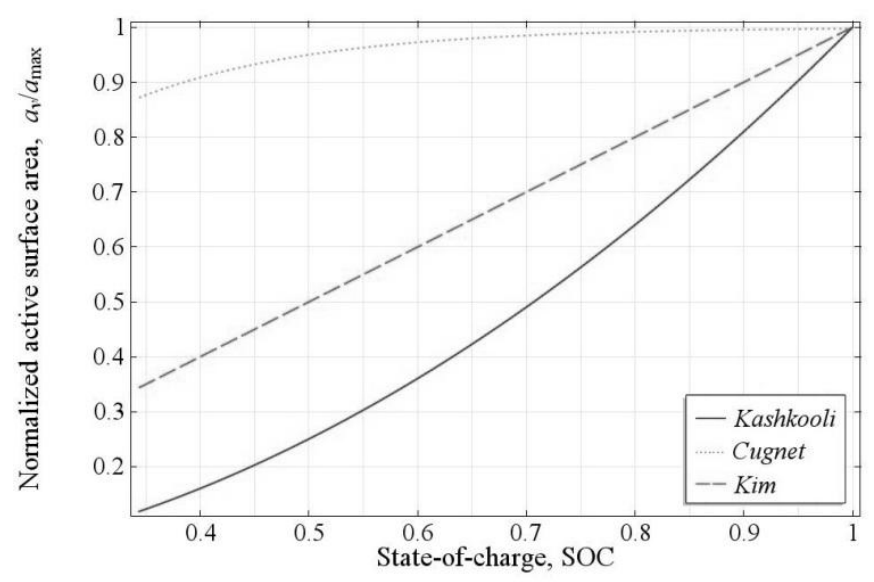

Fig. 8. Simulated morphology for POS during intermittent discharge over $20 \mathrm{~h}$ at $20^{\circ} \mathrm{C}$ using modified parameter values

\section{CONCLUSION}

Experiments indicate that the electrode morphology during discharge should be concave rather than convex (Tenno et al., 2001). The results clearly show that if the morphology parameter $\zeta$ is given a value smaller than 1 , the resulting morphology is concave. The morphology approximations by Cugnet are concave regardless of the value for $\zeta$ making it superior to the other approximation methods.

Also, the results clearly indicate that the SOC based on either porosity or charge per unit volume is very sensitive to the specified maximum value. Calculating the SOC independently of these specified maximum values is under investigation.

Other experiments indicate that the reactions at the EDL depend on the sulphuric acid concentration in a nonlinear fashion (Ekdunge and Simonsson, 1985). This means that the reaction order $\gamma$ should not be zero. The authors of Cugnet seem to compensate for this with the inclusion of an empirical parameter $\rho_{d}$. It seems that the authors of Kashkooli do not compensate for giving $\gamma$ a value of zero in any way. 
The current work is a step towards reducing confusion surrounding the complex processes in the EDL (Goodenough et al., 2007). With a detailed understanding about why so many lead-acid batteries still fail, better charge controllers and battery management systems are possible. Accurate active surface area approximations can be used in modelbased charge controllers as part of an overall healthconscious battery management system. This improvement will ensure that the end-user has no opportunity for incorrect and abusive operation of the battery.

Acknowledgements - The authors wish to thank HySA Infrastructure for funding this research.

\section{LIST OF SYMBOLS}

\begin{tabular}{|c|c|c|}
\hline Symbol & Name & Unit \\
\hline$a_{\max }$ & Maximum active surface area & $\mathrm{cm}^{2} / \mathrm{cm}^{3}$ \\
\hline$\alpha_{\mathrm{a}}$ & Anodic transfer coefficient & \\
\hline$\alpha_{\mathrm{c}}$ & Cathodic transfer coefficient & \\
\hline$C_{\mathrm{dl}}$ & Double-layer capacitance & $\mathrm{F} / \mathrm{cm}^{2}$ \\
\hline$c_{\text {ref }}$ & Reference concentration & $\mathrm{mol} / \mathrm{cm}^{3}$ \\
\hline$D_{l}$ & Diffusion constant of electrolyte & $\mathrm{cm}^{2} / \mathrm{s}$ \\
\hline ex & Tortuosity correction in liquid phase & \\
\hline exm & Tortuosity correction in solid phase & \\
\hline$\varepsilon_{0}$ & Porosity at zero charge & \\
\hline$\varepsilon_{\max }$ & Porosity at full charge & \\
\hline$F$ & Faraday constant of $96,485.3365$ & $(\mathrm{~A} \cdot \mathrm{s}) / \mathrm{mol}$ \\
\hline$\gamma$ & Reaction order & \\
\hline$i$ & Current density & $\mathrm{A} / \mathrm{cm}^{2}$ \\
\hline$i_{0}$ & Transfer current density & $\mathrm{A} / \mathrm{cm}^{2}$ \\
\hline$\kappa$ & Electrolyte conductivity & $\mathrm{S} / \mathrm{cm}$ \\
\hline $\mathrm{MW}_{\mathrm{M}}$ & Molecular weight of material M & $\mathrm{g} / \mathrm{mol}$ \\
\hline$\eta$ & Electrode overpotential & $\mathrm{V}$ \\
\hline$\phi$ & Electric potential & $\mathrm{V}$ \\
\hline$Q$ & Charge per unit volume & $\mathrm{C} / \mathrm{cm}^{3}$ \\
\hline$R$ & Universal gas constant of 8.3143 & $\mathrm{~J} /(\mathrm{mol} \cdot \mathrm{K})$ \\
\hline$\rho_{\mathrm{M}}$ & Density of material $\mathrm{M}$ & $\mathrm{g} / \mathrm{cm}^{3}$ \\
\hline$\sigma_{\mathrm{M}}$ & Conductivity of material $\mathrm{M}$ & $\mathrm{S} / \mathrm{cm}$ \\
\hline$T_{\text {cell }}$ & Cell temperature & $\mathrm{K}$ \\
\hline$t$ & Time & $\mathrm{s}$ \\
\hline$\zeta$ & Morphology parameter & \\
\hline
\end{tabular}

\section{REFERENCES}

Andersson, A. (2006). Battery Lifetime Modelling, Riso National Laboratory, Denmark.

Bernardi, D. M. and Carpenter, M. K. (1995). A Mathematical Model of the Oxygen-Recombination Lead-Acid Cell. Journal of The Electrochemical Society, 142 (8), The Electrochemical Society, p.2631-2642.

Bindner, H., Cronin, T., Lundsager, P., Manwell, J. F., Abdulwahid, U. and Baring-Gould, I. (2005). Lifetime Modelling of Lead Acid Batteries.

Bode, H. (1977). Lead-Acid Batteries. John Wiley \& Sons, New York.

Codecà, F., Savaresi, S. M. and Rizzoni, G. (2008). On battery state of charge estimation: A new mixed algorithm. In: IEEE International Conference on Control Applications , p.102-107.
Cugnet, M., Laruelle, S., Grugeon, S., Sahut, B., Sabatier, J., Tarascon, J.-M. and Oustaloup, A. (2009). A Mathematical Model for the Simulation of New and Aged Automotive Lead-Acid Batteries. Journal of The Electrochemical Society, 156 (12), The Electrochemical Society, p.A974-A985.

DeAnda, M. F., Miller, J., Moseley, P. and Butler, P. (2004). Reliability of Valve-Regulated Lead-Acid Batteries for Stationary Applications. (SAND2004-0914).

Ekdunge, P. and Simonsson, D. (1985). Recharge Kinetics of the Porous Lead Dioxide Electrode II. The Effect of Sulfuric Acid Concentration. Journal of The Electrochemical Society, 132 (11), The Electrochemical Society, p.2529-2533.

Goodenough, J. B., Abruña, H., Buchanan, M., Visco, S., Whittingham, M., Dunn, B., Gogotsi, Y., Gewirth, A., Nocera, D., Kelley, R. and others. (2007). Basic Research Needs for Electrical Energy Storage. US Department of Energy Report.

Gu, H., Nguyen, T. and White, R. E. (1987). A Mathematical Model of a Lead-Acid Cell: Discharge, Rest, and Charge. Journal of the Electrochemical Society, 134 (12), The Electrochemical Society, p.2953-2960.

Ibrahim, H., Ilinca, A. and Perron, J. (2008). Energy storage systems - Characteristics and comparisons. Renewable and Sustainable Energy Reviews, 12 (5), Elsevier, p.1221-1250.

Jongerden, M. R. and Haverkort, B. R. (2008). Battery Modeling. Centre for Telematics and Information Technology, University of Twente.

Kaiser, R. (2007). Optimized battery-management system to improve storage lifetime in renewable energy systems. Journal of Power Sources, 168 (1), Elsevier, p.58-65.

Kashkooli, A. G., Farhad, S., Fung, A. S. and Chen, Z. (2013). Effect of Convective Mass Transfer on LeadAcid Battery Performance. Electrochimica Acta, Elsevier.

Kim, S. C. and Hong, W. H. (1999). Analysis of the discharge performance of a flooded lead/acid cell using mathematical modelling. Journal of Power Sources, 77 (1), Elsevier, p.74-82.

Liu, R. S., Zhang, L., Sun, X., Liu, H. and Zhang, J. (2012). Electrochemical Technologies for Energy Storage and Conversion. Wiley-VCH, Germany.

Newman, J. and Tiedemann, W. (1975). Porous-Electrode Theory with Battery Applications. AIChE Journal, 21 (1), Wiley Online Library, p.25-41.

Ng, K., Moo, C., Lin, Y., Hsieh, Y. and Tsai, Y. (2007). Intermittent Discharging for Lead-Acid Batteries. In: Power Conversion Conference, Nagoya, p.216-220.

Nguyen, T., White, R. E. and Gu, H. (1990). The Effects of Separator Design on the Discharge Performance of a Starved Lead-Acid Cell. Journal of The Electrochemical Society, 137 (10), The Electrochemical Society, p.29983004.

Pavlov, D. (2011). Lead-Acid Batteries: Science and Technology. Elsevier Science.

Tenno, A., Tenno, R. and Suntio, T. (2001). Chargedischarge behaviour of VRLA batteries: model calibration and application for state estimation and failure detection. Journal of Power Sources, 103 (1), Elsevier, p.42-53.

Tiedemann, W. and Newman, J. (1975). Maximum Effective Capacity in an Ohmically Limited Porous Electrode. Journal of The Electrochemical Society, 122 (11), The Electrochemical Society, p.1482-1485.

Whitaker, S. (1986). Flow in Porous Media I: A Theoretical Derivation of Darcy's Law. Transport in Porous Media, 1 (1), Springer, p.3-25. 